\title{
Neutron-multiplicity experiments for enhanced fission modelling
}

\author{
Ali Al-Adili1, a , Diego Tarrío ${ }^{1}$, Franz-Josef Hambsch ${ }^{2}$, Alf Göök ${ }^{2}$, Kaj Jansson ${ }^{1}$, Andreas Solders ${ }^{1}$, \\ Vasileios Rakapoulos ${ }^{1}$, Cecilia Gustavsson ${ }^{1}$, Mattias Lantz ${ }^{1}$, Andrea Mattera ${ }^{1}$, Stephan Oberstedt ${ }^{2}$, \\ Alexander V. Prokofiev ${ }^{1}$, Erik A. Sundén ${ }^{1}$, Marzio Vidali ${ }^{2}$, Michael Österlund ${ }^{1}$, and Stephan Pomp ${ }^{1}$ \\ 1 Division of Applied Nuclear Physics, Department of Physics and Astronomy Uppsala University, Uppsala, Sweden \\ 2 European Commission, Joint Research Centre, Directorate G, Geel, Belgium
}

\begin{abstract}
The nuclear de-excitation process of fission fragments (FF) provides fundamental information for the understanding of nuclear fission and nuclear structure in neutron-rich isotopes. The variation of the prompt-neutron multiplicity, $\bar{v}(A)$, as a function of the incident neutron energy $\left(\mathrm{E}_{\mathrm{n}}\right)$ is one of many open questions. It leads to significantly different treatments in various fission models and implies that experimental data are analyzed based on contradicting assumptions. One critical question is whether the additional excitation energy $\left(\mathrm{E}_{\text {exc }}\right)$ is manifested through an increase of $\bar{v}(A)$ for all fragments or for the heavy ones only. A systematic investigation of $\bar{v}(A)$ as a function of $\mathrm{E}_{\mathrm{n}}$ has been initiated. Correlations between prompt-fission neutrons and fission fragments are obtained by using liquid scintillators in conjunction with a Frisch-grid ionization chamber. The proof-of-principle has been achieved on the reaction ${ }^{235} \mathrm{U}\left(\mathrm{n}_{\mathrm{th}}, \mathrm{f}\right)$ at the Van De Graff (VdG) accelerator of the JRC-Geel using a fully digital data acquisition system. Neutrons from ${ }^{252} \mathrm{Cf}(\mathrm{sf})$ were measured separately to quantify the neutron-scattering component due to surrounding shielding material and to determine the intrinsic detector efficiency. Prelimenary results on $\bar{v}(A)$ and spectrum in correlation with FF properties are presented.
\end{abstract}

\section{Introduction}

Improved determination of fission yields in correlation with particle-emission characteristics (prompt- neutrons and $\gamma$-rays) enhances the modeling of fission, provides input for nuclear structure studies and nuclear astrophysical processes, and could lead to more efficient and safe use of nuclear reactors and transmutation of nuclear waste. Presently, nuclear data libraries lack data, especially on the evolution of fission observables as a function of excitation energy $\left(\mathrm{E}_{\text {exc }}\right)$. The necessity of those data is evident for GEN IV reactors and for the scrutiny of sophisticated model calculations. In this work we focus on the prompt fission neutron multiplicity, $\bar{v}(A)$, as a function of $\mathrm{E}_{\mathrm{exc}}$ and especially how the fragment mass-dependence manifests itself in $\bar{v}\left(\mathrm{~A}, \mathrm{E}_{\mathrm{n}}\right)$. A handful earlier experiments supports a heavy-fragment extra-neutron emission but are surprisingly ignored in some contemporary fission calculation codes. More nuclear data are certainly needed.

This paper presents our first experimental efforts addressing this topic. The strategy is to make use of the double-E method in conjunction with liquid scintillators similarly to Ref. [1]. The main study case is the thermal neutron induced fission of ${ }^{235} \mathrm{U}$ by using a thermalised Van De Graaf fast neutron beam. Secondly, ${ }^{252} \mathrm{Cf}(\mathrm{sf})$ was measured during the same campaign as a bonus to be able to test our setup performance against the similar setup used in Ref. [1]. The latter measurement also serves for investigating the effect of neutron scattering (with and without used shielding) and to allow for a neutron energy

a e-mail: ali.al-adili@physics.uu.se calibration by use of the Mannhart evaluation. This paper shows the preliminary ${ }^{252} \mathrm{Cf}$ results as a proof-of-principle. The ${ }^{235} \mathrm{U}$ data are still being analysed but the provisional sawtooth shapes are reasonably compatible with to earlier literature data. In the near future we aim at measuring ${ }^{235} \mathrm{U}$ at $5.5 \mathrm{MeV}$ neutron energy and to compare the changes in the neutron multiplicity shape to the present data.

\section{Experiments}

The experimental setup, at JRC-Geel, is drawn schematically in Fig. 1. A Frisch-Grid Ionization Chamber (FGIC) was used together with two liquid scintillators (NE213 equivalent) (the used neutron detectors (ND) were characterised by Kornilov et al. [2]). Figure 2 shows one of the few existing measurements on the so-called "sawtooth" shape at different incident neutron energies $\left(E_{n}\right)$ [3]. The data implies that the extra neutron emission observed at higher $\mathrm{E}_{\mathrm{exc}}$ emerges mainly from the heavier fragments. Probably, the limited number of available data have implied that many fission codes as well as experimental data analysis, are based on an average increase instead. This was discussed in Refs. [4,5]. Particularly in the 2-E data this assumption has been shown to have significant effect on the measured mass yields [6]. To answer the data-needs the Uppsala group and JRC-Geel have initiated a measurement campaign.

The first experimental study cases were ${ }^{252} \mathrm{Cf}(\mathrm{sf})$ and ${ }^{235} \mathrm{U}\left(\mathrm{n}_{\mathrm{th}}, \mathrm{f}\right)$. During the beam-measurements at the VdG of JRC-Geel, two different U samples where used respectively $\left(0.1 \mathrm{mg} / \mathrm{cm}^{2}\right.$ and $\left.0.2 \mathrm{mg} / \mathrm{cm}^{2}\right)$. All samples are thin enough to allow for both fragments to escape. The

(c) The Authors, published by EDP Sciences. This is an Open Access article distributed under the terms of the Creative Commons Attribution License 4.0 (http://creativecommons.org/licenses/by/4.0/). 


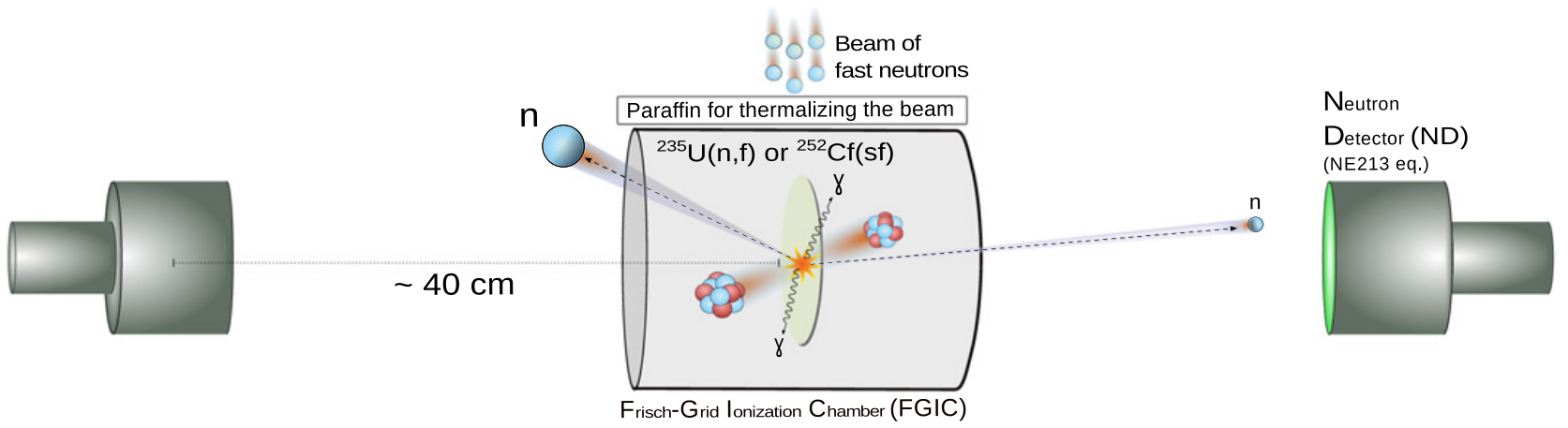

Figure 1. The experimental setup is schematically shown here, featuring the Frisch-Grid Ionization Chamber (FGIC) and the two liquid scintillators.

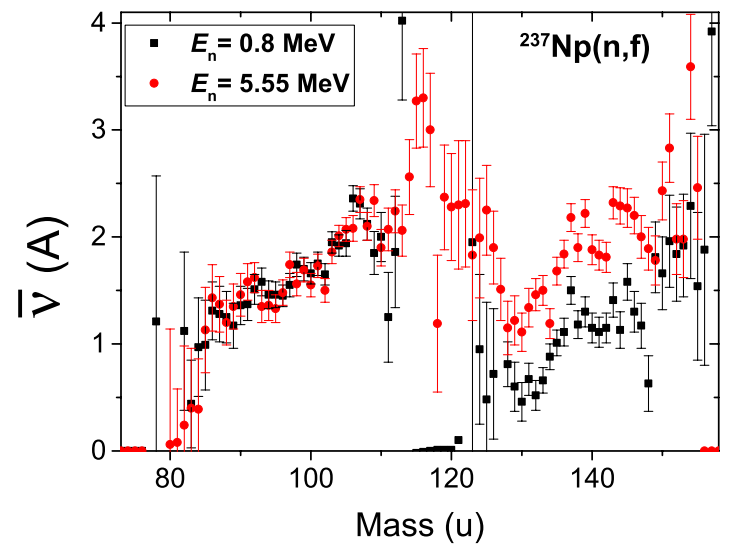

Figure 2. The $\bar{v}(A)$ for ${ }^{237} \mathrm{~Np}(\mathrm{n}, \mathrm{f})$ at different $\mathrm{E}_{\mathrm{n}}[3]$. The data shows a higher $\bar{v}$ but seemingly only from the heavy fragments.

neutron detectors were shielded from the beam by a wall of paraffin $+\mathrm{Pb}+\mathrm{Cu}$ sheets. The distances of the detectors to the fission source were $389 \mathrm{~mm}$ (Detector JRC-3 [2], right in picture) and $375 \mathrm{~mm}$ (Detector JRC-5, left in picture, facing the sample side of the FGIC).

For the ${ }^{235} \mathrm{U}$ case, a $0.5 \mathrm{MeV}$ neutron beam was produced via the ${ }^{7} \mathrm{Li}(\mathrm{p}, \mathrm{n})$ reaction. The neutrons were then moderated to thermal energies by a block of paraffin (about $12 \mathrm{~cm}$ ) put in front of the FGIC. The vast majority of acquired fission events were from thermal neutron induced fission due to the nature of the fission cross section and the moderated most neutron spectrum. Based on FLUKA simulations, the thermal neutron flux at the target was about $10^{4} \mathrm{n} / \mathrm{cm}^{2} \mathrm{~s}$.

The measurement on ${ }^{252} \mathrm{Cf}(\mathrm{sf})$ served as a proof-ofprinciple and to provide a neutron spectrum calibration. In addition we measured the $\mathrm{Cf}$ neutron spectrum with and without paraffin shielding to allow for an investigation of scattering effects on $\bar{v}(A)$.

During the experiments, digital data acquisition systems were utilized to record every signal from the chamber and the neutron detectors (ND). For the cathode and the two ND, a $400 \mathrm{MHz}$ sampling frequency was chosen, whereas for the anodes and grids, $100 \mathrm{MHz}$ digitizers were used. The triggering system was set with a coincidence condition between the chamber and the ND. The total fission rate was measured using triggers only from the FGIC, without the coincidence requirement with the ND.

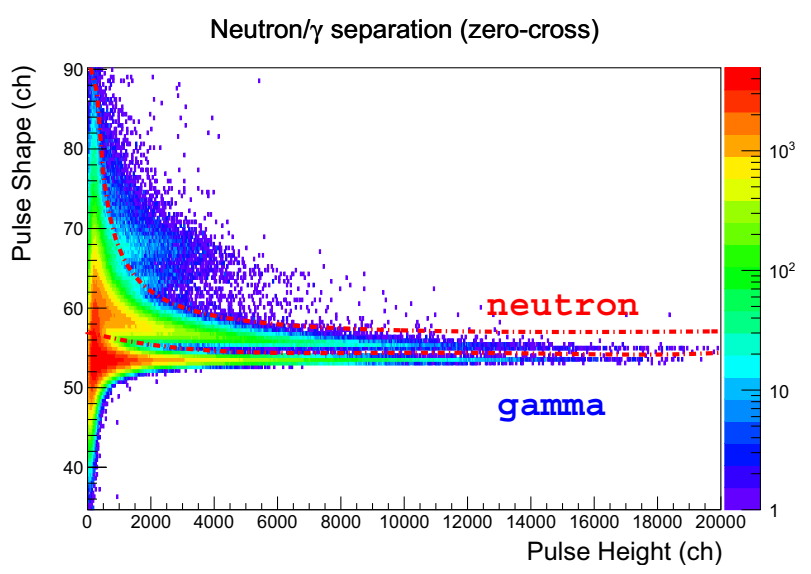

Figure 3. The pulse-shape discrimination is based on the zero-crossing method.

\section{Analysis}

The ${ }^{252} \mathrm{Cf}(\mathrm{sf})$ data are analyzed using the so-called 2-E technique where pre-neutron masses are iteratively calculated based on known prompt fission neutron multiplicities. The neutron multiplicity is calculated by the ratio of the two mass distributions, namely the coincidence and non-coincidence events (see Sect. 2) [1].

\subsection{Analysis of neutron data}

In order to extract the prompt fission neutrons, a pulseshape (PS) discrimination has been applied. Gaussian functions are fitted to both gamma and neutron PS peaks as a function of pulse height. Furthermore, cuts are made to the time-of-flight (TOF) based on the kinematics of the $(n, p)$ scattering in the liquid scintillator as well as a dynamical light output cut [7]. The TOF start and stop were derived from the FGIC cathode signal and the neutron detector signal, respectively. The timing resolution was about $\mathrm{FWHM}=1.5 \mathrm{~ns}$ as determined from the prompt fission gamma peak (Fig. 4). The final TOF of the fission neutrons is shown after the various data cuts in Fig. 4.

Once the measured neutron spectra are obtained, the internal detector efficiency has to be calculated. For that, the Mannhart evaluation was used (see Fig. 5) [8]. The measured neutron spectra are normalized to $\bar{v}_{t o t}=3.76$ and to the total number of fissions/measurement time. The solid angle coverage was in the order of $1 \%$ for the two detectors. The efficiency of the detectors are determined to be $40 \%$ around $1-2 \mathrm{MeV}$ neutron energy. 
Time-Of-Flight

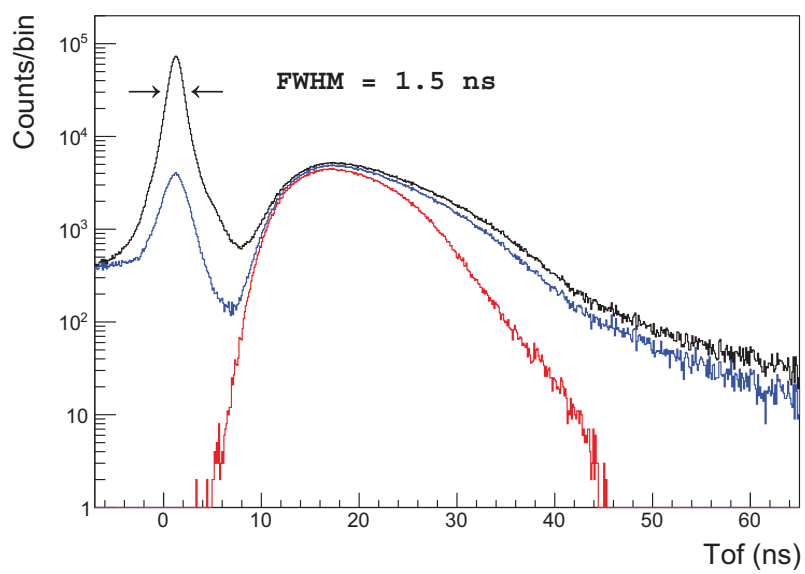

Figure 4. The time-of-flight of the neutrons between the cathode and the ND. The timing resolution is about $1.5 \mathrm{~ns}$ (raw spectrum in black). The effect of PS discrimination is seen on the TOF (blue). After the final TOF cut, the fission neutron contribution is drawn in red.

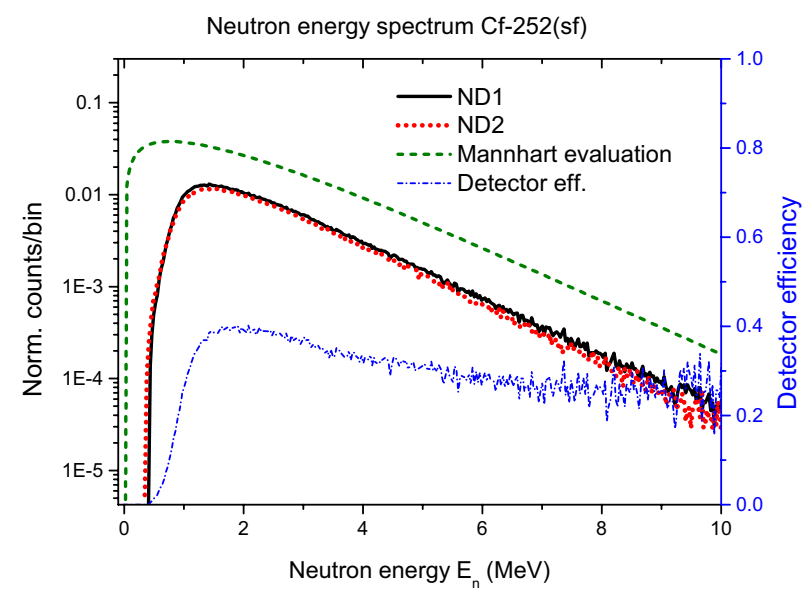

Figure 5. The ${ }^{252} \mathrm{Cf}$ neutron spectrum as measured in the two ND (full black and dotted red lines). The Mannhart evaluation (dashed green line) is used to estimate the internal detector efficiency (blue dash-dots, right axis) [8].

The detectors have a rather high threshold at $\mathrm{E}_{\mathrm{n}}=0.7 \mathrm{MeV}$, but this is no problem for this type of experiments $[1,9]$. Since the neutrons are assumed to be emitted from fullyaccelerated fragments, only neutrons above the average fragment energy/nucleon need to be considered.

\subsection{Analysis of fission fragment (FF) data}

The digital traces from the anodes and grids are treated, as in Ref. [10], to obtain the FF energies and angles. The data are corrected for grid inefficiency $[11,12]$ and baseline fluctuations. The FF angular distributions are obtained via the grid method [13]. The $\bar{v}(A)$ of Ref. [1] was used to calculate the pre-neutron emission distributions.

\section{Preliminary results and outlook}

The preliminary results presented here are from ${ }^{252} \mathrm{Cf}(\mathrm{sf})$ for which the analysis has proceeded most. The preneutron mass distribution from ${ }^{252} \mathrm{Cf}(\mathrm{sf})$ is shown in Fig. 6 compared to the data of Ref. [1]. The agreement is rather
Pre-neutron Mass Cf-252 (2E method)

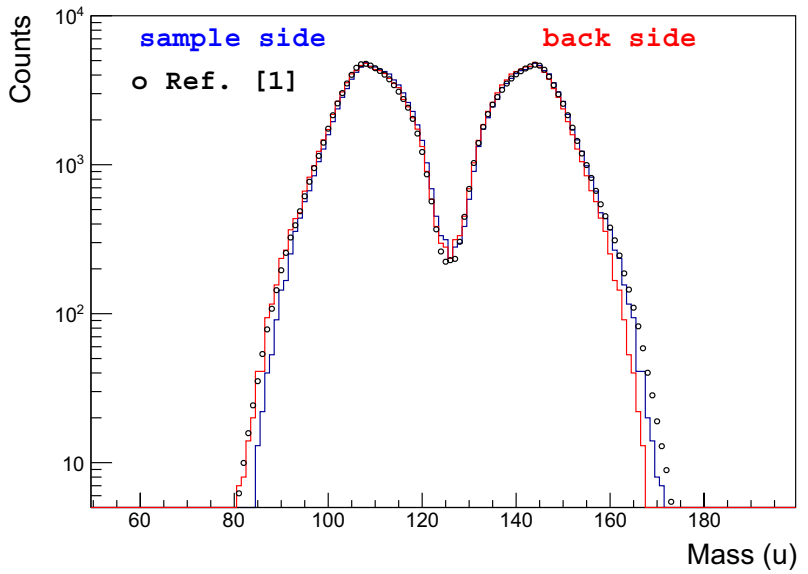

Figure 6. The $\mathrm{Y}(\mathrm{A})$ of ${ }^{252} \mathrm{Cf}(\mathrm{sf})$ from the 2 -E technique. The two chamber sides are compared to Ref. [1].

\section{TKE vs Mass Cf-252(sf)}

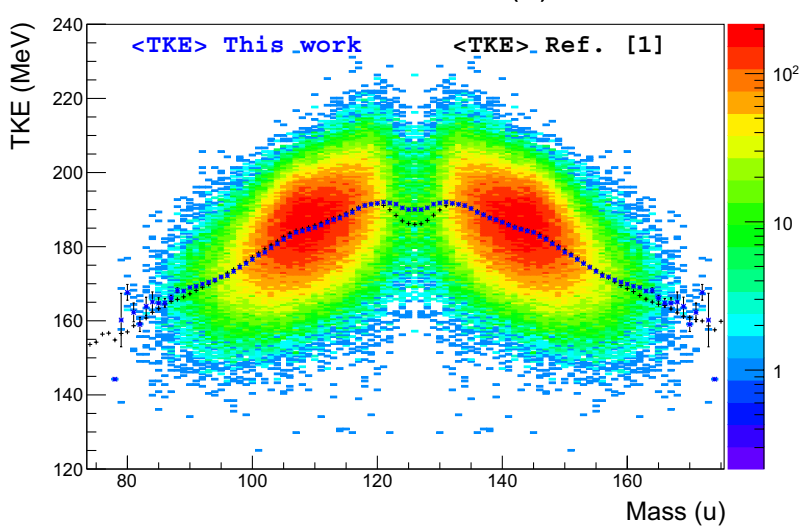

Figure 7. The TKE versus mass for ${ }^{252} \mathrm{Cf}(\mathrm{sf})$ distribution together with the average values compared to Ref. [1].

satisfactory although the analysis is not finalized yet. Some small differences are present at the outer mass-distribution wings, probably attributed to problems in the angular distributions.

The Total Kinetic Energy (TKE) distribution versus mass is also plotted in Fig. 7 together with the mean TKE values [1]. The results are in good agreement except at symmetry. The reason for the discrepancy is unclear up to this point, but will probably be resolved once the analysis steps are refined (angular determination and energy loss corrections).

The provisional mass $(\mu)$ distributions, are calculated from the ratio of detected energies (corrected for energy losses and pulse-height defect) and are shown in Fig. 8. They differ from the final pre-neutron masses since no iterative procedure has been applied yet (including to account for the prompt neutron emission). At this moment the provisional $\bar{v}(\mu)$ distribution is determined from the ratio of those two provisional (coincidence and noncoincidence) mass distributions. The data are compared to $\bar{v}(\mathrm{~A})$ data from Ref. [1] in Fig. 9. The analysis is currently being finalized to obtain pre-neutron emission masses after the iterative procedure, and to obtain $\bar{v}(A)$ after applying the corrections mentioned in Ref. [1]. In doing so it is necessary to make an initial assumption of $\bar{v}(\mathrm{~A})$ in order to obtain the pre-neutron masses. Figure 9 shows that the 


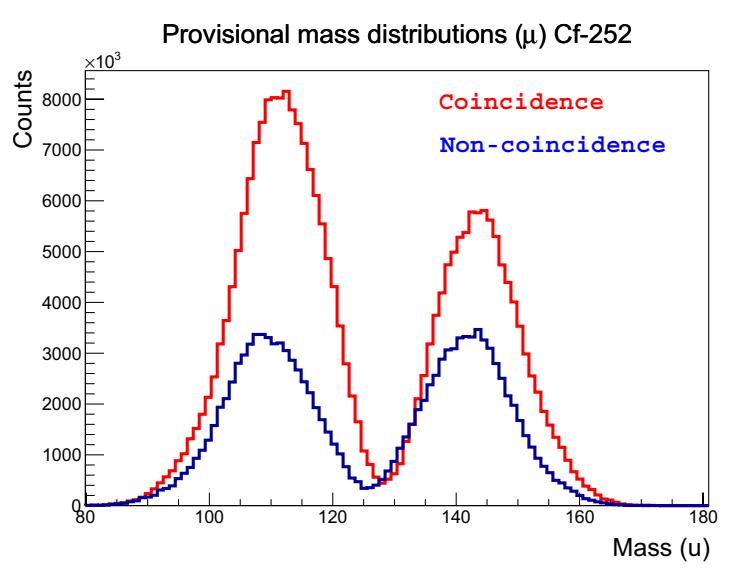

Figure 8. The provisional mass distributions, from the ratio of the detected fragment energies. The non-coincidence (blue), the coincidence data between the ND and the chamber (red).

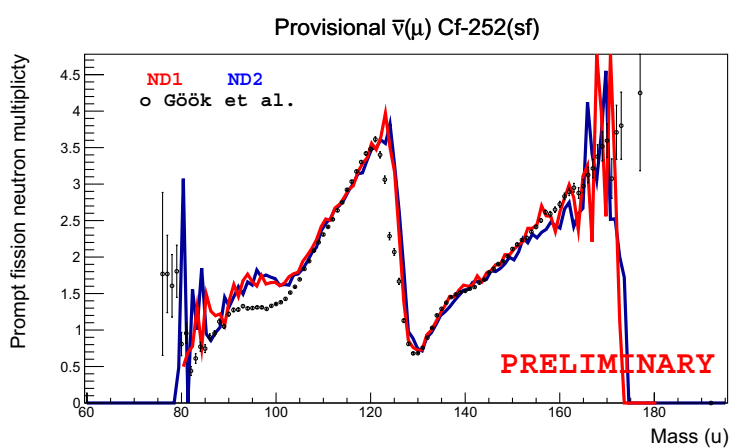

Figure 9. The ratio of the two distributions of Fig. 8 gives the provisional saw-tooth shape of ${ }^{252} \mathrm{Cf}(\mathrm{sf})$ [1].

impact of the initial assumption made on the $\bar{v}(\mathrm{~A})$ on the final result will be small.

Finally, we are planning for a measurement of ${ }^{235} \mathrm{U}\left(\mathrm{n}_{\mathrm{th}}, \mathrm{f}\right)$ at $E_{n}=5.5 \mathrm{MeV}$. In addition, the study of the neutron data from ${ }^{252} \mathrm{Cf}(\mathrm{sf})$ allows us to study systematic effects such as the impact of the paraffin shielding (measured both with and without the shielding environment) and the effect of using a thicker Uranium target (also used during beam time) on the final sawtooth shape.

This project has been financed by the Swedish Radiation Safety authority (SSM). This work has also been supported by the CHANDA trans-national access program and the JRC-EUFRAT program. We would like to thank the operators of the VdG of JRC-Geel.

\section{References}

[1] A. Göök et al., Phys. Rev. C 90, 064611 (2014)

[2] N.V. Kornilov et al., Nucl. Meth. Instr. A 599, 226 (2009)

[3] A. Naqvi et al., Phys. Rev. C34, 218 (1986)

[4] A. Al-Adili et al., Nucl. Data. Sheets 119, 342 (2014)

[5] A. Al-Adili et al., EPJ Web Conf. 122, 01007 (2016)

[6] A. Al-Adili et al., Phys. Rev. C86, 054601 (2012)

[7] N.V. Kornilov, Fission Neutrons, (SPRINGER 2015), ISBN: 978-3-319-07132-9

[8] W. Mannhart, Proc. Advisory Group Meeting on Properties of Neutron Sources, Leningrad, USSR, 913 June 1986 (IAEA, Vienna, 1987), p. 158

[9] C. Budtz-Jørgensen and H.-H. Knitter, Nucl. Phys. A 490, 307 (1988)

[10] A. Al-Adili et al., Nucl. Meth. Instr. A 624, 684 (2010)

[11] A. Al-Adili et al., Nucl. Meth. Instr. A673, 116 (2012)

[12] A. Göök et al., Nucl. Meth. Instr. A664, 103 (2012)

[13] A. Al-Adili et al., Nucl. Meth. Instr. A671, 103 (2012) 